\title{
Asthma affects time to pregnancy and fertility: a register-based twin study
}

\author{
Elisabeth J. Gade ${ }^{1,2}$, Simon F. Thomsen 1 , Svend Lindenberg ${ }^{2}$, Kirsten O. Kyvik ${ }^{3}$, \\ Sofie Lieberoth ${ }^{1}$ and Vibeke Backer ${ }^{1}$
}

Affiliations: ${ }^{1}$ Respiratory Research Unit, Bispebjerg University Hospital, Copenhagen, ${ }^{2}$ Copenhagen Fertility Center, Copenhagen, and ${ }^{3}$ The Danish Twin Registry, Institute for Health and Service Research, Odense, Denmark.

Correspondence: E.J. Gade, Respiratory Research Unit, Bispebjerg University Hospital, Bispebjerg Bakke 23, DK-2400 Copenhagen NV, Denmark. E-mail: Elisabeth_gadedyahoo.com

ABSTRACT Coexistence of infertility and asthma has been observed clinically. Therefore, we investigated the association between asthma and delayed pregnancy in a nationwide population-based cohort of twins.

A cohort of 15250 twins living in Denmark (aged 12-41 years) participated in a questionnaire study including questions about the presence of asthma and fertility. Differences in time to pregnancy and pregnancy outcome were analysed in subjects with asthma, allergy and in healthy individuals using multiple regression analysis.

Asthma was associated with an increased time to pregnancy, the percentage of asthmatics with a time to pregnancy $>1$ year was $27 \%$ versus $21.6 \%$ for non-asthmatics (OR (95\% CI) $1.31(1.1-1.6) ; \mathrm{p}=0.009)$. The association remained significant after adjustment for age, age at menarche, body mass index and socioeconomic status (OR (95\% CI) $1.25(1.0-1.6) ; \mathrm{p}=0.05)$, and was more pronounced in those $>30$ years of age $(32.2 \%$ versus $24.9 \%$, OR (95\% CI) $1.44(1.1-1.9) ; \mathrm{p}=0.04)$. Untreated asthmatics had a significant increased risk of prolonged time to pregnancy compared to healthy individuals (OR (95\% CI) 1.79 $(1.20-2.66) ; \mathrm{p}=0.004)$, while asthmatics receiving any kind of treatment for asthma tended to have a shorter time to pregnancy than untreated asthmatics (OR 1.40; $\mathrm{p}=0.134$ ).

Asthma prolongs time to pregnancy. The negative effect of asthma on fertility increases with age and with disease intensity, indicating that a systemic disease characterised by systemic inflammation also can involve reproductive processes.

@ERSpublications

Asthma prolongs time to pregnancy: the negative effect of asthma on fertility increases with age and disease intensity http://ow.ly/qpa9B

A press release for this article is available from www.erj.ersjournals.com/site/misc/presspack.xhtml

Received: Aug 262013 | Accepted after revision: Oct 082013 | First published online: Nov 142013

Support statement: This study was supported by grants from FAPS (Union of Practicing Specialists) (grant number 29241/11), Lundbeck Pharmaceutical and the Medical Association Denmark (grant number R100-A9502).

Conflict of interest: None declared.

Copyright @ERS 2014 


\section{Introduction}

Asthma is one of the most common chronic diseases in females of reproductive age, occurring in up to $8 \%$ of pregnancies [1]. Approximately $24 \%$ of all couples that attempt to conceive will be infertile for one or more period lasting for $>1$ year [2], and 3-6\% of 45-year-old females are involuntarily infertile and have never given birth [3].

Coexistence of asthma and infertility is, therefore, present in a large group of patients. Several studies have shown interplay between the severity of asthma and female reproductive health, including menstrual cycle, abortions and pregnancy, although the cause of this association is poorly understood [4-6]. Moreover, it has been suggested that oestrogen has an impact on the aetiology of chronic inflammatory/autoimmune disease [7], but whether oestrogen is a friend or foe in patients with chronic inflammatory diseases remains controversial [8]. The effects of asthma on fertility remain poorly investigated and, at present, there is only little evidence to suggest a connection between asthma and infertility.

A recent study by CARSON et al. [9] suggests an association between subfertility, fertility treatment and asthma in children born through in vitro fertilisation (IVF). This may be due to the IVF treatment itself but could also be due to a genetic predisposition to asthma. The last assumption suggests that a greater proportion of asthmatic parents seek fertility treatment, indicating a link between asthma and infertility. This assumption seems credible since a study by KäLLÉN et al. [10] reported that a greater amount of females undergoing IVF treatment were using anti-asthmatic drugs.

Furthermore, it is well known that patients with other chronic inflammatory and autoimmune diseases (e.g. rheumatoid arthritis and Sjögren's disease) have a prolonged time to pregnancy (TTP) and a reduction in fertility [11, 12]. Autoimmune conditions may affect all stages of fertility such as ovarian failures, implantation failure and pregnancy loss [13].

In contrast to the above, TATA et al. [14] found similar fertility rates (live births per 1000 person-years) in females with allergic disease (asthma, eczema and hay fever) compared with females from the general population. The study also found that females with hay fever or eczema were more fertile than those without these conditions. Furthermore, asthma was hypothesised to have a different relationship to fertility than eczema and hay fever, as young asthmatic individuals had a higher fertility rate than their older counterparts compared with the healthy group.

Overall, the association between asthma and infertility is not fully resolved. Accordingly, we investigated whether asthmatic patients experience longer waiting TTP. To examine this, we conducted a twin-based cohort study to estimate population-based TTP and perinatal outcomes in females with asthma compared with females without asthma.

\section{Material and methods}

Study population

The study population comprised all female twins born between 1953 and 1982 who were enrolled in the Danish Twin Registry [15]. Data from the Danish Twin Registry were used due to its large size, its detailed questionnaire and the ability to compare twin sisters with respect to pregnancy outcomes controlling for unmeasured confounding variables such as genetics and lifestyle factors. Our aim was to generalise the results to the singleton population, which has been shown to be possible [16]. A total of 15250 females participated in a nationwide questionnaire study concerning general health and lifestyle. The study also included questions about asthma and fertility. The age of the participants ranged between 12 and 41 years (mean age 26.8 years) and the participation rate in the general health study was $86 \%$. In total 7979 females did not respond to questions about their fertility. The nonresponders were younger than responders $(22.3$ versus 32.0 years), which clearly indicates a tendency for younger females not to respond as to whether they have not been pregnant or had attempted to conceive yet. In contrast, nonresponding was not related to asthma status and, hence, responding was only associated with our outcome not the exposure, thereby minimising bias due to nonresponding.

\section{Asthma and allergy}

Asthma cases were identified on the basis of an affirmative response to the question "Have you ever had asthma?" This procedure has been shown to be reliable for excluding subjects without asthma in population-based studies, which use questionnaire responses as the sole diagnostic criterion. Tóren et al. [17] validated the diagnosis of asthma by questionnaires in relation to a clinical diagnosis of asthma; the mean sensitivity for the question about self-reported asthma was $68 \%$ in the reviewed studies, while the specificity was $94 \%$. Allergy cases were identified on the basis of an affirmative response to the question: "Have you ever had allergy?" 


\section{Medical history}

The asthmatic patients were divided into treated and non-treated asthmatics based on two questions in the questionnaire. The questions used were: "I used or have previously used asthma medication (yes/no)?" And "Do you use, or have you ever used any medication during longer periods on a regular basis or due to attacks?" The females, who responded to the second question that they received medication in the category "medicine to treat obstructive pulmonary disease" were considered as treated asthmatics.

Furthermore the treated asthmatic group was subdivided into a group that received inhaled corticosteroids daily and a group that only received reliever medication. Thereby, we were able to assess whether the degree of treatment had an impact on TTP.

\section{Fecundity measures}

We evaluated the lifetime prevalence of infertility, which indicates the prevalence of all who are infertile at the time of the survey and/or who had been infertile for one or more period in their lives. Lifetime prevalence of infertility was identified on the basis of an affirmative response to the question: "Have you and your partner ever spent more than 1 year trying to become pregnant without pregnancy occurring within this year?" Conception was defined as achievement of a clinical pregnancy. Abnormal TTP was censored after 12 months because the clinical definition of infertility is defined as 1 year of unprotected intercourse in the fertile phase of the menstrual cycle without conception [18].

TTP has been proven to be a valuable tool to measure fecundity; it is easy to obtain information about and is well recalled even many years after pregnancy. TTP is influenced not only by biological factors, but also knowledge and behaviour. TTP as an outcome measure has been extensively used in epidemiological studies aiming to identify the effects of, for example, adverse lifestyle or changes in fertility over time [19].

The mean number of offspring was calculated based on the average amount of biological children each female had given birth to.

We examined pregnancy outcome in order to assess whether the reason for a possible prolonged TTP could be due to more frequent adverse pregnancy outcomes. Pregnancy outcomes were identified on the basis of the question: "Was the outcome of your first pregnancy a live-born child, a stillbirth, miscarriage, abortion or ectopic pregnancy?"

\section{Statistical analysis}

Data were analysed with the statistical package SPSS version 20.0 (SPSS Inc., Chicago, IL, USA). Mean \pm SD were calculated for the normally distributed data, whereas median and interquartile ranges were used to describe the skewed distributed data. The groups were compared using the two-sample t-test for the normally distributed continuous variables and the non-parametric Mann-Whitney U-test for non-normally distributed continuous variables. Fisher's exact test was used for categorical, unpaired data. Values of $\mathrm{p}<0.05$ were considered significant.

First, the association between asthma and TTP was analysed in the whole cohort $(n=15250)$ with asthma (yes or no) as the dependent variable. In multivariate analysis of regression, we explored the potentially confounding effects of age, smoking, body mass index (BMI), age at menarche and socioeconomic status (years of education).

A 1:1 matched conditional logistic regression analysis (co-twin control analysis) was used to examine the effect of asthma on TTP in twin pairs discordant for asthma. The matching was carried out with the asthmatic twin in each pair being the case and the non-asthmatic twin being the control. In the co-twin control analysis, a higher risk of long TTP in dizygotic (DZ) co-twins compared with monozygotic (MZ) co-twins in relation to asthma would indicate an underlying genetic relationship between asthma and TTP, whereas a higher risk of long TTP in MZ co-twins compared with DZ co-twins would indicate an underlying non-genetic relationship between asthma and TTP [20].

\section{Results}

Of 15250 respondents, 955 (6.3\%) reported a history of asthma. The distribution of females with asthma according to age, smoking, BMI, age at menarche and educational level is shown in table 1 . The distribution of covariates amongst asthmatic individuals and non-asthmatic individuals was similar, with the exception of BMI, which was significantly higher in the asthmatic group $\left(22.24\right.$ versus $\left.21.66 \mathrm{~kg} \cdot \mathrm{m}^{-2} ; \mathrm{p}<0.001\right)$, and age at menarche, which was significantly younger in the asthmatic group (13.07 versus 13.18 years; $\mathrm{p}=0.029)$.

The proportion of females whose TTP was prolonged was significantly higher in the asthmatic group compared with the non-asthmatic group (27\% versus $21.6 \%$, OR (95\% CI) $1.31(1.07-1.61) ; \mathrm{p}=0.009)$ 
TABLE 1 Characteristics of the study population

\begin{tabular}{|c|c|c|c|c|c|c|}
\hline & Total cohort & Healthy & Asthmatic & $\begin{array}{c}\text { Prevalence of } \\
\text { asthma }\end{array}$ & $\begin{array}{c}\text { Mean difference } \\
(95 \% \mathrm{Cl})\end{array}$ & p-value \\
\hline Subjects & $15250^{\#}$ & 14011 & 955 & & & \\
\hline Age group years & & & & & & 0.547 \\
\hline $12-17$ & 2425 & $2276(16.2)$ & $149(15.6)$ & 6.1 & & \\
\hline $18-25$ & 3937 & $3671(26.2)$ & $266[27.9]$ & 6.8 & & \\
\hline $26-30$ & 3187 & 2989 (21.3) & $198(20.7)$ & 6.2 & & \\
\hline $31-35$ & 2935 & $2751(19.6)$ & $184(19.3)$ & 6.3 & & \\
\hline $36-41$ & 2482 & $2324(16.6)$ & $158(16.5)$ & 6.4 & & \\
\hline Current smoking & $4608(30.9)$ & 4309 (30.9) & $299(31.4)$ & 6.5 & $1.02(0.89-1.18)^{+}$ & 0.773 \\
\hline Body mass index $\mathrm{kg} \cdot \mathrm{m}^{-2}$ & $21.69 \pm 3.7$ & $21.66 \pm 3.7$ & $22.24 \pm 4.7$ & & $0.58(0.27-0.89)$ & $<0.001$ \\
\hline Years of education ${ }^{\pi}$ & & & & & & 0.556 \\
\hline 0 & 374 & $345(2.5)$ & $29(3.0)$ & 7.8 & & \\
\hline $1-9$ & 2703 & $2515(18.0)$ & $188(19.7)$ & 7.0 & & \\
\hline $10-13$ & 8597 & 8060 (57.5) & $537(56.2)$ & 6.2 & & \\
\hline $13-15$ & 3048 & $2869(20.5)$ & $179(18.7)$ & 5.9 & & \\
\hline $16-25$ & 242 & $2.20(1.6)$ & 22 (2.3) & 9.1 & & \\
\hline Age at menarche years & $13.17 \pm 1.4$ & $13.18 \pm 1.4$ & $13.07 \pm 1.5$ & 6.4 & $-0.105(-0.20--0.011)$ & 0.029 \\
\hline Age at conception years & $24.49 \pm 5.3$ & $24.54 \pm 5.3$ & $23.74 \pm 5.7$ & 6.3 & $0.80(0.4-0.90)$ & $<0.001$ \\
\hline Age at first birth years & $26.14 \pm 4.2$ & $26.19 \pm 4.2$ & $25.49 \pm 4.2$ & 6.7 & $0.70(0.30-1.12)$ & 0.001 \\
\hline
\end{tabular}

(table 2). The association remained significant after adjustment for age, BMI and smoking (1.27 $(1.01-1.59) ; \mathrm{p}=0.04)$. Additional adjustment for age at menarche and socioeconomic status led to a further small decrease in risk $(1.25(1.00-1.58) ; \mathrm{p}=0.05)$. Restricting the analysis to females $>30$ years of age showed an even stronger tendency towards long waiting TTP amongst asthmatic individuals (32.2 versus $24.9 \%, 1.44$ (1.10-1.88); $\mathrm{p}=0.04$ after adjustment for confounders).

We investigated whether suffering from allergies only or suffering from both asthma and allergy had an influence on fertility (table 3). Individuals with allergy had the same TTP as the general population $(21.2 \%$ versus $21.6 \%$, OR $(95 \% \mathrm{CI}) 0.91(0.76-1.10))$. Females with both allergy and asthma had a slight, but not significant, prolonged TTP compared with the general population ( $26.5 \%$ versus $21.6 \%$, OR (95\% CI) 1.28 $(0.96-1.72))$. The increased risk of prolonged TTP among females with asthma and allergy was comparable to being asthmatic alone with a decreased odds ratio (26.5\% versus $27 \%$ ) (table 3 ). Therefore, allergy is not significantly associated with changes in TTP.

Females with asthma were found to conceive earlier in life than those without (23.74 versus 24.53 years; $\mathrm{p}<0.001$ ) and were consequently younger when giving birth for the first time ( 25.49 versus 26.19 years; $\mathrm{p}=0.001)$ (table 1$)$. The mean number of offspring in the two groups was similar ( 1.86 versus 1.83 children; $p=0.509)$ and the proportion of childless females was identical in the two groups $(6.5 \%$ versus $6.2 \% ; \mathrm{p}=0.355)$ (table 2$)$.

TABLE 2 The relationship between asthma and prolonged time to pregnancy (TTP)

\begin{tabular}{lcccc} 
& Non-asthmatic & Asthmatic & OR (95\% CI) & p-value \\
\hline TTP >1 year & $1415(21.6)$ & $120(27.0)$ & $1.3(1.07-1.61)$ & $\mathbf{0 . 0 0 9}$ \\
Adjusted, 1 step & & $1.27(1.01-1.59)$ & $\mathbf{0 . 0 4}$ \\
Adjusted, 2 step & & $1.25(1.0-1.58)$ & 0.05 \\
Temporarily infertile & & & $0.81(0.53-1.21)$ & 0.355 \\
Childless $^{\mathbf{5}}$ & $1003(15.4)$ & $88(20.2)$ & $1.02(0.99-1.05)$ & 0.552 \\
\hline
\end{tabular}

Data are presented as $\mathrm{n}(\%)$, unless otherwise stated. Statistically significant $\mathrm{p}$-values are shown in bold. \#: adjusted for age, body mass index (BMI) and smoking; ": adjusted for age, BMI, smoking, age at menarche and educational status; ${ }^{+}$: proportion of females that have conceived, despite an infertile period in their life; s: proportion of females from the total cohort who never, despite attempts to, conceived. 
TABLE 3 The association between prolonged time to pregnancy (TTP), allergy and asthma

\begin{tabular}{|c|c|c|c|c|c|}
\hline & TTP $>1$ year & OR $(95 \% \mathrm{CI})$ & p-value & Adjusted OR $(95 \% \mathrm{Cl})^{\#}$ & Adjusted $\mathrm{p}$-value ${ }^{\#}$ \\
\hline Healthy & $1438(21.6)$ & 1.00 & & & \\
\hline Allergy & $194(21.2)$ & $0.95(0.82-1.10)$ & 0.522 & $0.91(0.76-1.10)$ & 0.30 \\
\hline
\end{tabular}

Data are presented as $\mathrm{n}(\%)$, unless otherwise stated. " : adjusted for age, body mass index, smoking, age at menarche and educational status.

The sub-fertile group (TTP $>1$ year) (asthmatics and healthy cohort) differed from the fertile groups (TTP $<1$ year) with respect to several descriptive variables (table 4). They had a higher prevalence of obesity (higher BMI), were older, had a shorter education and a greater proportion were smokers. There were no differences in these variables between the subjects with asthma and the healthy group.

Untreated asthmatics had a significantly increased risk of prolonged TTP compared with healthy individuals ( $30.5 \%$ versus $21.6 \%$, OR $1.79 ; \mathrm{p}=0.004)$, while asthmatics receiving any kind of treatment for asthma trended to have a shorter TTP than untreated asthmatics $(23.8 \%$ versus $30.5 \%$, OR $1.40 ; \mathrm{p}=0.134)$. Asthmatics receiving daily-inhaled corticosteroid treatment also showed increased risk of prolonged TTP compared with healthy subjects $(33.0 \%$ versus $21.6 \%$, OR $2.34 ; \mathrm{p}=0.003)$ (table 5$)$.

There were no significant differences between those with asthma and those without regarding stillbirths, extrauterine pregnancies and spontaneous abortions. However, there was a tendency towards increased risk of miscarriage in the asthmatic group (12.3\% versus $9.8 \%$, OR $1.27(0.93-1.80)$ ). This effect was not statistically significant $(\mathrm{p}=0.169)$ (table 6).

Co-twin control analysis in twin pairs discordant for asthma showed no significant difference in the risk of prolonged TTP in the asthmatic compared with the non-asthmatic twin (OR 0.29, $\mathrm{p}=0.12$ in MZ twins and OR $0.77, p=0.53$ in DZ twins). However, only 80 twin pairs were included in this analysis: $26 \mathrm{MZ}$ twin pairs and 54 DZ twin pairs. The risk of long TTP was not significantly different in DZ co-twins compared with MZ co-twins, indicating a direct adverse effect of asthma on TTP not mediated through genetic or nongenetic confounding factors.

\section{Discussion}

This study showed that TTP is significantly prolonged in asthmatic individuals even after adjusting for basic variables such as BMI, age and smoking. The impact of asthma on TTP is consistent in older and younger individuals, with an increasing tendency with age. This finding supports the findings of TATA et al. [14], who showed that females with asthma tend to have lower fertility rates when older compared with healthy females of similar age.

It strengthens our findings that those with asthma, in general, tend to conceive at an earlier age compared to those without, which in theory should make them more fertile than the healthy group. There seems to be no obvious explanation for this observation.

Untreated asthmatics have a significantly increased risk of prolonged TTP compared to healthy individuals. Furthermore, TTP seems to be reduced in asthmatics receiving treatment for their asthma compared with non-treated asthmatics, but being treated for asthma does not negate the shown effect completely compared with healthy subjects. However, when dividing the treatment groups into subgroups, our data show that asthmatics who received inhaled corticosteroids also have an excess risk compared with healthy individuals.

TABLE 4 The association between time to pregnancy (TTP) and the descriptive variables

\begin{tabular}{lcccc} 
& TTP $<1$ year & TTP $>\mathbf{1}$ year & Mean difference (95\% Cl) & $\mathbf{p}$-value \\
\hline Subjects $\mathbf{n}$ & 5468 & 1561 & & \\
Age years & $31.7 \pm 5.6$ & $33.3 \pm 4.7$ & $1.6(1.30-1.90)$ & $<.001$ \\
Body mass index $\mathbf{~ k g} \cdot \mathbf{m}^{-2}$ & $22.4 \pm 3.7$ & $22.7 \pm 4.1$ & $0.3(0.1-0.5)$ & $\mathbf{0 . 0 1}$ \\
Years of education & $11.0 \pm 2.4$ & $10.6 \pm 2.3$ & $-0.4(-0.5--0.2)$ & $<0.001$ \\
Current smoking & $2058(37.2)$ & $733(47.0)$ & $1.5(1.34-1.68)$ & $<0.001$ \\
Age at menarche years & $13.25 \pm 1.4$ & $13.26 \pm 1.5$ & $0.01(-0.09-0.08)$ & 0.899 \\
\hline
\end{tabular}

Data are presented as mean \pm SD or $n(\%)$, unless otherwise stated. Statistically significant $p$-values are shown in bold. ${ }^{\#}:$ mean \pm SEM. 
TABLE 5 The effect of asthma medication on time to pregnancy (TTP)

\begin{tabular}{lccc} 
Medication & Prolonged TTP & OR (95\% Cl) & p-value \\
\hline $\begin{array}{l}\text { Self-reported medication } \\
\text { Non-asthma }\end{array}$ & $1410(21.6)$ & & $<0.001$ \\
Untreated & $60(30.5)$ & $1.79(1.20-2.66)$ & 0.004 \\
Any treatment & $65(23.8)$ & $1.13(0.85-1.51)$ & 0.389 \\
$\quad$ Corticosteroids & $37(33.0)$ & $2.34(1.33-4.13)$ & 0.003 \\
$\quad$ Other treatments & $28(17.4)$ & $0.76(0.51-1.15)$ & 0.199 \\
Medication question & & & \\
Treated & $71(26.0)$ & 1.00 & 0.198 \\
Untreated & $20(34.5)$ & $1.13(0.93-1.28)$ & \\
\hline
\end{tabular}

Data are presented as $\mathrm{n}(\%)$, unless otherwise stated. ${ }^{\#}$ : prolonged TTP was defined as TTP $>1$ year; ": calculated on the basis of the question: "Do you or have you ever used any medication during longer periods on a regular basis or due to attacks?"; ${ }^{+}$: calculated on the basis of the question: "I'm using or have previously used asthma medication".

This could indicate that the negative effect of asthma on fertility increases with the severity of the disease, as asthmatics in need of corticosteroids are suffering from moderate-to-severe asthma. Another explanation, which should not be excluded, is that treatment with inhaled corticosteroids could be the cause of the prolonged TTP. This does not seem obvious since the increased risk of prolonged TTP for the corticosteroidtreated asthmatics did not differ from the untreated asthmatics compared to the healthy group.

Based on our results, one could assume that the prolonged TTP among asthmatics could be explained by the increased tendency for spontaneous abortion in this group. Although we must be cautious to conclude on a trend that is not significant, it is worth noting that the risk of spontaneous abortions in asthmatics seems to be increased by $27 \%$. Furthermore, this trend is consistent with recent results from BLAIS et al. [5], who showed an increased risk of spontaneous abortions amongst asthmatics (OR (95\% CI) 1.41 (1.33-1.49)), which was independent of the severity of the asthmatic disease.

Our results are in line with the knowledge available about the effect of asthma on pregnancy. Studies have shown that asthma influences the fetus during pregnancy by increasing the risk of abortions, preterm birth, low birth weight and perinatal mortality. Conversely, well-medicated asthma during pregnancy improves intrauterine growth of the fetus and lowers adverse perinatal outcomes [21]. This tendency also seems to be the case when attempting to become pregnant, exemplified by our observation.

Our data show that the number of offspring is identical between asthmatics and healthy subjects, consistent with the findings of TATA et al. [14]. However, KARMAUS et al. [22] showed that atopic individuals tend to have fewer children. Interestingly, while the waiting TTP in those with asthma is longer, they ultimately have the same number of children as those without. This may be partly explained by the fact that they try to become pregnant at an earlier age, but other unknown factors may also play a role.

The above suggests that asthma has a major impact on fertility and pregnancy and that this effect may be reduced by treatment. With this in mind, it seems natural to assume that the systemic inflammation that characterises asthma may account for some of the effect of asthma on infertility.

TABLE 6 Pregnancy outcome in a cohort of asthmatics and non-asthmatics

\begin{tabular}{lccc} 
Pregnancy outcome & Non-asthmatics & Asthmatics & OR (95\% Cl) \\
\hline Total pregnancy outcomes ${ }^{\#}$ & $5224(91.9)$ & $349(6.2)$ & $1(0.25-2.6)$ \\
Live births & $4313(82.6)$ & $281(80.5)$ & 0.548 \\
Twins & $70(1.3)$ & $3(0.9)$ & 0.685 \\
Stillbirths & $57(1.1)$ & $3(0.9)$ & 0.680 \\
Spontaneus abortions & $512(9.8)$ & $43(12.3)$ & $1.27(0.93-1.8)$ \\
Extrauterine pregnancy & $53(1.0)$ & $6(1.3)$ & 0.445 \\
Induced abortions & $57(1.1)$ & $12(3.4)$ & 0.169 \\
Pregnant & $162(3.1)$ & 0.247 & 0.500 \\
\hline
\end{tabular}

Data are presented as $\mathrm{n}(\%)$, unless otherwise stated. ${ }^{*}$ : outcomes that could not be affected by asthma during pregnancy were not included. 
In both animal and human studies, systemic inflammation in asthma has been shown to affect organs other than the respiratory system, particularly the brain, the cardiovascular system (altered vascular reactivity) and the blood (altered cytokine profile in plasma) [23]. An explanation for our findings, which would be in accordance with this, could be a change in the blood supply of the uterus, or an increased infiltration of inflammatory cells into the mucosal layer in the uterus. In terms of fertility, an altered blood supply or an increased infiltration of inflammatory cells into the endometrial lining could lead to impaired implantation or even rejection of the fertilised egg.

This assumption is supported by the findings of PONNOTH et al. [24], who showed that systemic inflammation caused by asthma in mice could alter the vascular reactivity in the body (impaired vasorelaxation in response to adenosine). In line with this, others have shown an increased risk of cardiovascular disorders in asthmatic patients [25]. The role of systemic inflammation in those with asthma, regarding both fertility and other organs in the body, is still unclear and further research is needed to understand the mechanisms that link asthma with its comorbidities [26].

The apparently increased negative impact of asthma on fertility with age may be explained by prolonged exposure to the systemic inflammation with age. If the systemic inflammation that characterises asthma is the cause of the observed impact on the fertility a treatment option for fertile asthmatic patients could be of a more systemic nature, such as leukotriene receptor antagonists or other targeted therapies.

With regards to inflammation and asthma, it has been indicated that there might be a common inflammatory pathway between endometriosis and asthma, as well as between the metabolic syndrome and asthma. In terms of endometriosis, females that suffer from this disease have a higher prevalence of asthma and allergy than the general population [27]. Furthermore, leukotriene receptor antagonists, intended for asthma treatment, have been found to be useful in the treatment of endometriosis [28]. There is also growing evidence that the metabolic syndrome (abdominal obesity, insulin resistance, hypertension and dyslipidaemia) is a strong risk factor for asthma, even stronger than obesity [29]. A study by VAN HUISSTEDE et al. [30] showed a correlation between eosinophilia, lung function and metabolic syndrome, while no correlation was observed for BMI. The possible causal connections between endometriosis and asthma, and the metabolic syndrome and asthma could be some of the explanatory factors behind the impaired fertility of asthma patients, as both diseases are known to reduce fertility [31, 32].

There are some limitations to this study. One is possible recall bias as our data were obtained from selfadministered questionnaires. Validation studies have shown that self-reported TTP in questionnaires gives an accurate representation of the TTP distribution [33, 34], even with longer recall periods of up to 20 years [35].

Another possible bias is lack of generalisability to the singleton population as a whole. It is well known that twin pregnancies are at increased risk for pre-eclampsia, preterm birth and being small for gestational age $[36,37]$. Furthermore, it has been suggested that children born with low birth weight and to a mother with pre-eclampsia are at a greater risk of asthma [38], although the evidence is circumstantial [39, 40]. A possible higher proportion of asthmatics in our dataset than in the general population would, however, not affect the relationship with TTP. Moreover, the proportion of asthmatics in our cohort was 6.3\%, which corresponds well with estimates from the general population (6.6\%) [41]. Unfortunately we had no data on birth weight and, therefore, we were not able to take this into account in our analyses.

Despite these issues, twins can be assumed to be representative of the population because they are born into all social groups and their morbidity/mortality after infancy is equivalent to the mortality of the general population [42]. Therefore, they can be analysed as individuals epidemiologically. Furthermore, studies of twins have shown that twins represent the fecundity of the general population [43].

Another limitation to this study is incomplete information about fertility-related disease and the severity of asthma. Furthermore, it would have been interesting to have information on whether the participants had sought fertility treatment or if their partner had reduced fertility. It was not possible, based on the present data, to determine whether asthmatics seek fertility treatment more frequently than healthy individuals, which, based on our findings, we might expect.

Lastly, a large number of participants did not respond to questions about their fertility. This group was significantly younger than the other respondents. Therefore, we assume that they had not used their fertility potential at the time of the questionnaire. This could be considered as a selection bias, but analysis of regression shows that the dropouts are representative of the whole cohort in terms of asthma status, smoking and BMI. Accordingly, the large number of dropouts does not seem to be critical to our results. Furthermore, when we restricted the analysis to females $>30$ years of age, in whom the response rate to the fertility questions was high, the negative effect of asthma on TTP was even more pronounced, indicating that, at worst, the lack of response diluted our estimate. 
In conclusion, asthmatics experience a longer TTP, but ultimately achieve the same average number of children. These findings seem contradictory, but those with asthma were also found to have an earlier start to their reproductive life, leading to a similar number of offspring. These findings could be related to systemic inflammation, as our data indicate that receiving asthma medication reduces TTP, but further evidence is needed for confirmation.

\section{Acknowledgements}

Special thanks to L.R. Skadhauge (Dept of Occupational Medicine, Hospital of South West Jutland, Denmark) for contributing to the data collection and design of the questionnaire.

\section{References}

1 Hansen C, Joski P, Freiman H, et al. Medication exposure in pregnancy risk evaluation program: the prevalence of asthma medication use during pregnancy. Matern Child Health J 2013; 17: 1611-1621.

2 Slama R, Hansen OKH, Ducot B, et al. Estimation of the frequency of involuntary infertility on a nation-wide basis. Hum Reprod 2012; 27: 1489-1498.

3 Schmidt L, Münster K, Helm P. Infertility and the seeking of infertility treatment in a representative population. $\mathrm{Br}$ J Obstet Gynaecol 1995; 102: 978-984.

4 Svanes C, Real FG, Gislason T, et al. Association of asthma and hay fever with irregular menstruation. Thorax 2005; 60: 445-450.

5 Blais L, Kettani FZ, Forget A. Relationship between maternal asthma, its severity and control and abortion. Hum Reprod 2013; 28: 908-915.

6 Sheiner E, Mazor M, Levy A, et al. Pregnancy outcome of asthmatic patients: a population-based study. J Matern Fetal Neonatal Med 2005; 18: 237-240.

7 Østensen $\mathrm{M}$, Brucato A, Carp $\mathrm{H}$, et al. Pregnancy and reproduction in autoimmune rheumatic diseases. Rheumatology (Oxford) 2011; 50: 657-664.

8 Townsend EA, Miller VM, Prakash YS. Sex differences and sex steroids in lung health and disease. Endocr Rev 2012; 33: $1-47$.

9 Carson C, Sacker A, Kelly Y, et al. Asthma in children born after infertility treatment: findings from the UK Millennium Cohort Study. Hum Reprod 2013; 28: 471-479.

10 Källén B, Otterblad Olausson P. Use of anti-asthmatic drugs during pregnancy. 1. Maternal characteristics, pregnancy and delivery complications. Eur J Clin Pharmacol 2007; 63: 363-373.

11 Jawaheer D, Zhu JL, Nohr EA, et al. Time to pregnancy among women with rheumatoid arthritis. Arthritis Rheum 2011; 63: 1517-1521.

12 Hussein SZ, Jacobsson LTH, Lindquist PG, et al. Pregnancy and fetal outcome in women with primary Sjögren's syndrome compared with women in the general population: a nested case-control study. Rheumatology (Oxford) 2011; 50: 1612-1617.

13 Carp HJA, Selmi C, Shoenfeld Y. The autoimmune bases of infertility and pregnancy loss. J Autoimmun 2012; 38: J266-J274.

14 Tata LJ, Hubbard RB, McKeever TM, et al. Fertility rates in women with asthma, eczema, and hay fever: a general population-based cohort study. Am J Epidemiol 2007; 165: 1023-1030.

15 Kyvik KO, Christensen K, Skytthe A, et al. The Danish Twin Register. Dan Med Bull 1996; 43: 467-470.

16 Andrew T, Hart DJ, Snieder H, et al. Are twins and singletons comparable? A study of disease-related and lifestyle characteristics in adult women. Twin Res 2001; 4: 464-477.

17 Torén K, Brisman J, Järvholm B. Asthma and asthma-like symptoms in adults assessed by questionnaires. A literature review. Chest 1993; 104: 600-608.

18 Evers JL. Female subfertility. Lancet 2002; 360: 151-159.

19 Joffe M, Villard L, Li Z, et al. A time to pregnancy questionnaire designed for long term recall: validity in Oxford, England. J Epidemiol Community Health 1995; 49: 314-319.

20 Duffy DL. The co-twin control study. In: Spector TD, Snieder H, MacGregor AJ, eds. Advances in Twin and SibPair Analysis. London, Greenwich Medical Media Ltd., 2000.

21 Rocklin RE. Asthma, asthma medication and their effects on maternal/fetal outcomes during pregnancy. Reprod Toxicol 2011; 32: 189-197.

22 Karmaus W, Eneli I. Maternal atopy and the number of offspring: is there an association? Pediatr Allergy Immunol 2003; 14: 470-474.

23 Jousilahti $\mathrm{P}$, Salomaa $\mathrm{V}$, Hakala $\mathrm{K}$, et al. The association of the sensitive systemic inflammation markers with bronchial asthma. Ann Allergy Asthma Immunol 2002; 89: 381-385.

24 Ponnoth DS, Nadeem A, Mustafa SJ. Adenosine-mediated alteration of vascular reactivity and inflammation in a murine model of asthma. Am J Physiol Heart Circ Physiol 2008; 294: 2158-2165.

25 Iribarren C, Tolstykh IV, Eisner MD. Are patients with asthma at increased risk of coronary heart disease? Int J Epidemiol 2004; 33: 743-748.

26 Cazzola M, Segreti A, Calzetta L, et al. Comorbidities of asthma: current knowledge and future research needs. Curr Opin Pulm Med 2013; 19: 36-41.

27 Matalliotakis I, Cakmak H, Matalliotakis M, et al. High rate of allergies among women with endometriosis. J Obstet Gynaecol 2012; 32: 291-293.

28 Mshemish BA, Sadik SM, Ashor AW. Studying the effect of montelukast in the treatment of dysmenorrhea: a single-blind, placebo-controlled trial. Mustansiriya Med J 2012; 11: 37-41.

29 Adeyeye OO, Ogbera AO, Ogunleye OO, et al. Understanding asthma and metabolic syndrome - a Nigerian report. Int Arch Med 2012; 5: 20.

30 van Huisstede A, Cabezas MC, Birnie E, et al. Systemic inflammation and lung function impairment in morbidly obese subjects with the metabolic syndrome. J Obes 2013; 2013: 131349. 
31 Usadi RS, Legro RS. Reproductive impact of polycystic ovary syndrome. Curr Opin Endocrinol Diabetes Obes 2012; 19: 505-511.

32 Barnhart K, Dunsmoor-Su R, Coutifaris C. Effect of endometriosis on in vitro fertilization. Fertil Steril 2002; 77: $1148-1155$.

33 Baird DD, Weinberg CR, Rowland AS. Reporting errors in time-to-pregnancy data collected with a short questionnaire. Impact on power and estimation of fecundability ratios. Am J Epidemiol 1991; 133: 1282-1290.

34 Zielhuis GA, Hulscher ME, Florack EI. Validity and reliability of a questionnaire on fecundability. Int J Epidemiol 1992; 21: 1151-1156.

Joffe M, Villard L, Li Z, et al. Long-term recall of time-to-pregnancy. Fertil Steril 1993; 60: 99-104.

Smith-Levitin M, Skupski DW, Chervenak FA. Multifetal pregnancies. Curr Opin Obstet Gynecol 1995; 7: 465-471. Fox NS, Roman AS, Saltzman DH, et al. Risk factors for preeclampsia in twin pregnancies. Am J Perinatol 2014; 31: 163-166.

38 Brooks AM, Byrd RS, Weitzman M, et al. Impact of low birth weight on early childhood asthma in the United States. Arch Pediatr Adolesc Med 2001; 155: 401-406.

39 Miyake Y, Tanaka K. Lack of relationship between birth conditions and allergic disorders in Japanese children aged 3 years. J Asthma 2013; 50: 555-559.

40 Yang HJ, Qin R, Katusic S, et al. Population-based study on association between birth weight and risk of asthma: a propensity score approach. Ann Allergy Asthma Immunol 2013; 110: 18-23.

41 Dodge RR, Burrows B. The prevalence and incidence of asthma and asthma-like symptoms in a general population sample. Am Rev Respir Dis 1980; 122: 567-575.

42 Öberg S, Cnattingius S, Sandin S, et al. Twinship influence on morbidity and mortality across the lifespan. Int J Epidemiol 2012; 41: 1002-1009.

43 Jensen TK, Joffe M, Scheike T, et al. Time trends in waiting time to pregnancy among Danish twins. Hum Reprod 2005; 20: 955-964. 